\title{
Hypoglycaemia in the early neonatal period in newborns with risk factors
}

\author{
Tatyana E Taranushenko ${ }^{1 *}$, Ekaterina V Antsiferova ${ }^{1,2}$ and Natalia G Kiseleva ${ }^{1}$ \\ ${ }^{1}$ Department of Pediatrics, Institute of Postgraduate Education, Krasnoyarsk State Medical University named after prof. Voino-Yasenetsky, Institute of Postgraduate \\ Education for Pediatricians and Neonatologists, Krasnoyarsk, Russia \\ ${ }^{2}$ Department of Newborns in Obstetric Hospital, Krasnoyarsk Regional Clinical Center for Maternity and Childhood Protection, Krasnoyarsk, Russia
}

\begin{abstract}
Neonatal hypoglycaemia is a topical problem in neonatology and paediatric endocrinology. In recent years, problems of glycaemia monitoring during first days of life have been discussed as well as the strategy for management of newborns with the syndrome of hypoglycemia and evaluation of metabolism in brain neurons has been a matter of scientific research. Scientific publications regarding the detection rate and duration of low glucose values in the early neonatal period as well as means of their correction and conducted therapy results are very scarce and ambiguous, which determines the necessity of new modern data regarding the problem considered. Purpose of the study: to study the detection rate and dynamics of clinical and laboratory manifestations of hypoglycaemia in children in the early neonatal period in order to optimise observation of newborns at the risk of developing this condition. Materials and methods: an open, retrospective, continuous, single-centre study was conducted to obtain preliminary data important for development of monitoring strategies for newborns at the risk of hypoglycaemia (neonates with high birth weight, children born to mothers with diabetes, newborns with intrauterine growth restriction). The target studied group was constituted by 522 newborns of both genders in the early neonatal period (aged 0 to 7 days). Results: the paper presents data on hypoglycaemia detection rate in newborns with risk factors for hypoglycaemia development, clinical and laboratory features of this condition, dynamics of glycaemia during the early neonatal period as well as the timing of blood glucose stabilisation (the age of normoglycaemia achievement).
\end{abstract}

\section{Introduction}

Hypoglycaemia in a newborn is reduction of glucose concentration in blood below $2.6 \mathrm{mmol} / \mathrm{l}$ in the neonatal period irrespective of the gestational age [1,2].

Neonatal hypoglycaemia is a topical problem in neonatology and paediatric endocrinology. In recent years, problems of glycaemia monitoring during first days of life have been discussed as well as the strategy for management of newborns with the syndrome of hypoglycemia and evaluation of metabolism in brain neurons has been a matter of scientific research [3].

Physiological immaturity of carbohydrate homeostasis is characteristic to the early neonatal period. Therefore, more than one half of neonates are unable to maintain the fasting blood glucose level within normal values. Further glucogenesis activation and enteral glucose consumption provide for normoglycaemia achievement by the end of the first day of life. Hypoglycaemia persisting beyond the aforementioned period is based on transient hyperinsulinism (diabetes mellitus in the pregnant patient, perinatal asphyxia), transient glucose deficiency (intrauterine growth restriction, premature birth) medicine intake by the pregnant mother and/or the newborn (angiotensinconverting enzyme inhibitors, valproic acid, indomethacin, etc.) as well as on a severe somatic pathology (sepsis, CHD, etc.) The above perinatal factors are predictors of transient impairments of carbohydrate metabolism in the early neonatal period. The highest probability of blood glucose level decrease in risk group children exists for 42 hours after birth and may persist for 2-3 days [4,5].

The peculiarity of neonatal hypoglycaemia is non-specificity of the clinical picture. Analogical manifestations may be associated with other diseases (sepsis, intraventricular haemorrhage, asphyxia, CHD, etc.). The criteria making it possible to reveal hypoglycaemia in newborns through differential diagnostics (Whipple's triad) suggest appearance of clinical symptoms against the background of low blood glucose levels, their disappearance after normalisation of the laboratory values and further reappearance in case of glycaemia decrease [6].

However, neonatal hypoglycaemia progresses asymptomatically in most cases, even against the background of substantial blood glucose level deviation below the lower reference value. There with, according to the literature, untimely correction of neonatal hypoglycaemia leads to various neurological impairments in postnatal life (psychomotor development retardation, lowered IQ, impairment of sensory and cognitive functions, behavioural disorders, etc. Several cohort studies carried out in different countries have demonstrated high frequency of minimal cerebral dysfunction in children with asymptomatic hypoglycaemia in medical history. Some authors point at the association of severe neonatal hypoglycaemia with development of convulsive disorder in the early childhood. These circumstances define the

${ }^{\star}$ Correspondence to: Tatyana E. Taranushenko, Department of Pediatrics, Institute of Postgraduate Education, Krasnoyarsk State Medical University named after prof. Voino-Yasenetsky, Institute of Postgraduate Education for Pediatricians and Neonatologists, Krasnoyarsk, Russia, Tel: +7 910 737-27-42; E-mail: tetar@rambler.ru

Key words: newborns, diabetic fetopathy, hypoglycemia in newborns, intrauterine growth restriction, high birth weight

Received: February 07, 2021; Accepted: February 15, 2021; Published: February 19,2021 
necessity of routine monitoring of blood glucose level during first days of life in children at the risk of compromised carbohydrate homeostasis [7-10].

Scientific publications regarding the detection rate and duration of low glucose values in the early neonatal period as well as means of their correction and conducted therapy results are very scarce and ambiguous, which determines the necessity of new modern data regarding the problem considered.

\section{Purpose of the study}

To study the occurrence rate and the dynamics of clinical and laboratory manifestations of hypoglycaemia in children in the early neonatal period in order to discuss possibilities in optimisation of observation for newborns at the risk of development of this condition.

\section{Materials and methods}

An open, retrospective, continuous, single-centre study aimed to acquire preliminary data important for planning of further observation strategy has been conducted. Results of observation of 522 neonates born in the Krasnoyarsk Regional Clinical Center for Maternity and Childhood Protection (KRCCMCP) in 2018 at the risk of hypoglycaemia development are presented in the paper. The risk factors were considered to be high birth weight, being born to a mother with diabetes mellitus, intrauterine growth restriction (IUGR). The studied groups were comparable in gender and age.

According to the existing protocol of management of newborns at the risk of hypoglycaemia development (a local normative document of the KRCCMCP based on international and Russian guidelines), the first blood glucose evaluation was performed no later than 1 hour after the birth with consequent evaluations every 3 hours during the first day of life, every 6 hours for the following three days, and 2 times per day starting from the 5 th day of life. Blood glucose monitoring ended at the blood glucose level of $2.6 \mathrm{mmol} / \mathrm{l}$ persisting for 24 hours against the background of enteral feeding and absence of intravenous glucose infusion. The Fenton Preterm Growth Chart for preterm neonates and WHO centile charts for term newborns were used to evaluate physical development of the subjects.

\section{Inclusion criteria}

Newborns with risk factors for hypoglycaemia development: high birth weight (with reference to the gestational age), diabetes mellitus in the mother, IUGR of the newborn.

\section{Exclusion criteria}

Newborns with no risk factors for hypoglycaemia development, children born out of the inpatient facility, children with congenital developmental defects of the gastrointestinal tract, severe perinatal impairments of the central nervous system, critical care in the early neonatal period.

Statistical data of the analyzed qualitative attributes were represented by absolute numbers and percentages. Quantitative attributes were presented using median values with distribution quartiles Me [Q1;Q2]. Statistical significance of the differences between attributes of the independent samples was evaluated via the Mann-Whitney test while differences between the qualitative attributes were evaluated using the Chi-square test. The differences between the attributes were considered significant at $\mathrm{p}<0.05$. Mathematical processing of the results obtained was carried out using a personal computer and software applications
MS Excel 2016 (Microsoft Corporation, USA), Statistica 8.0 (StatSoft Inc., USA).

This work was approved by the local ethics committee at the FSBEI HE Prof. V.F. Voino-Yasenetsky KrasSMU MOH Russia (summary of protocol No.53/2015).

\section{Results}

A total of 3721 children were born in the KRCCMCP within the analysed period. A total of 522 children were enrolled in the study (children at the risk of hypoglycaemia development) and randomised into 3 groups.

1. Group 1: 88 children with large body weight with reference to gestational age.

2. Group 2: 213 children born to mothers with diabetes mellitus.

3. Group 3: 221 children with intrauterine growth restriction.

Children with high birth weight were $17.0 \%$ of the subjects, hypoglycaemia was revealed in 24 newborns (27\%, Figure 1). Among these, $67 \%$ neonates were male and $33 \%$ were female $(\mathrm{p}=0.0005)$.

The median gestational age in the group was 38 weeks [38;39], the median body mass was $4280 \mathrm{~g}$ [4158;4443]. The proportion of children with a body mass within the $90-97$ centile corridor was $67 \%$. Accordingly, the body mass exceeded the 97 th centile in $33 \%$ of the cases. The minimum determined glycaemia values were $1.8 \mathrm{mmol} / \mathrm{l}$ [2.1;2.5]. Clinical manifestations of glycaemia in the studied group were absent (were not registered) in $96 \%$ of the cases (including the child with the lowest glycaemia level). However, $4 \%$ of the children were observed to have clinical manifestations of low blood glucose levels in the form of motor activity reduction (Figure 2).

The age distribution of newborns with consideration for the term of diagnosed hypoglycaemia was as follows. Low blood glucose levels were revealed in $88 \%$ of the cases during the 1 st day of life, $8 \%$ during the 2 nd day of life and $4 \%$ during the 3rd (Figure 3 ).

Upon evaluation of glycaemia indices, it was noted that $71 \%$ of the neonates had lowest blood glucose values during the first day of life, $25 \%$ of the children had the lowest newly diagnosed glycaemia during the 2 nd day of life while the same condition was observed during the 3 rd day of life in $4 \%$ of the cases. Depending on the glycaemia level, hypoglycaemia reversal therapy involved intravenous injection of $10 \%$ glucose (in $25 \%$ of all cases in the studied group), peroral administration of $5 \%$ glucose solution (71\%) supplementary feeding with breast milk or infant formula (4\%). Hypoglycaemia was reversed with no consequent relapse in $67 \%$ of the newborns during the 1st day of life, in $25 \%$ during the 2 nd day of life and in $8 \%$ during the 3 rd day of life.

Children born to mothers with diabetes mellitus amounted $40.8 \%$ in the studied cohort, the strong majority of these children were born to mothers with gestational diabetes mellitus (98\%), the proportion of females with diabetes mellitus type 1 was $2 \%(p=0.0005)$, diabetes mellitus type 2 was not registered in mothers from the studied group. Hypoglycaemia was registered in 95 of the newborns (44.6\%, Figure $1)$, the gender distribution did not reveal significant differences $(50.5 \%$ male, $49.5 \%$ female, $\mathrm{p}=0.5284$ ). The median gestational age was 38 weeks [36;39], median body mass was in this group of children was 3300 g [2900;3955]. The body mass was in compliance with the gestational age in $49.5 \%$ of the children, exceeded the 90 th centile in $46.3 \%$ of the cases and did not exceed the 10th centile in $4.2 \%$ of the newborns. The minimum glucose level in the studied group was $0.7 \mathrm{mmol} / 1[1.9 ; 2.4]$ 


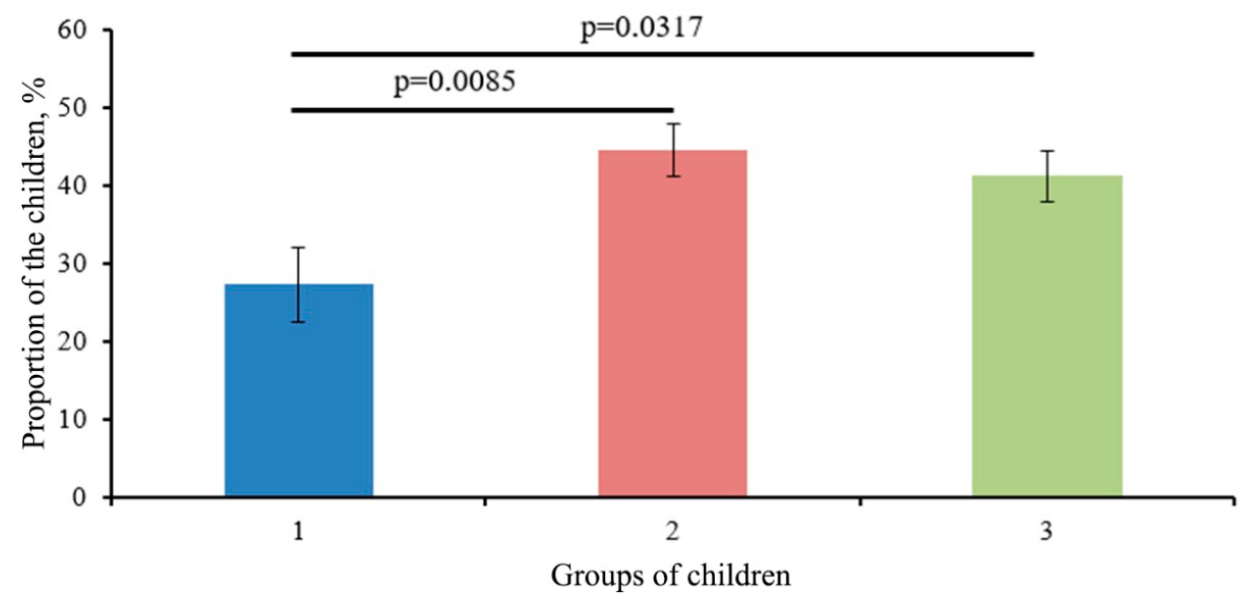

Figure 1. Frequency of detection of hypoglycemia in the groups. Note:*the assessment of statistical significance is presented according to the $\chi 2$ calculation.

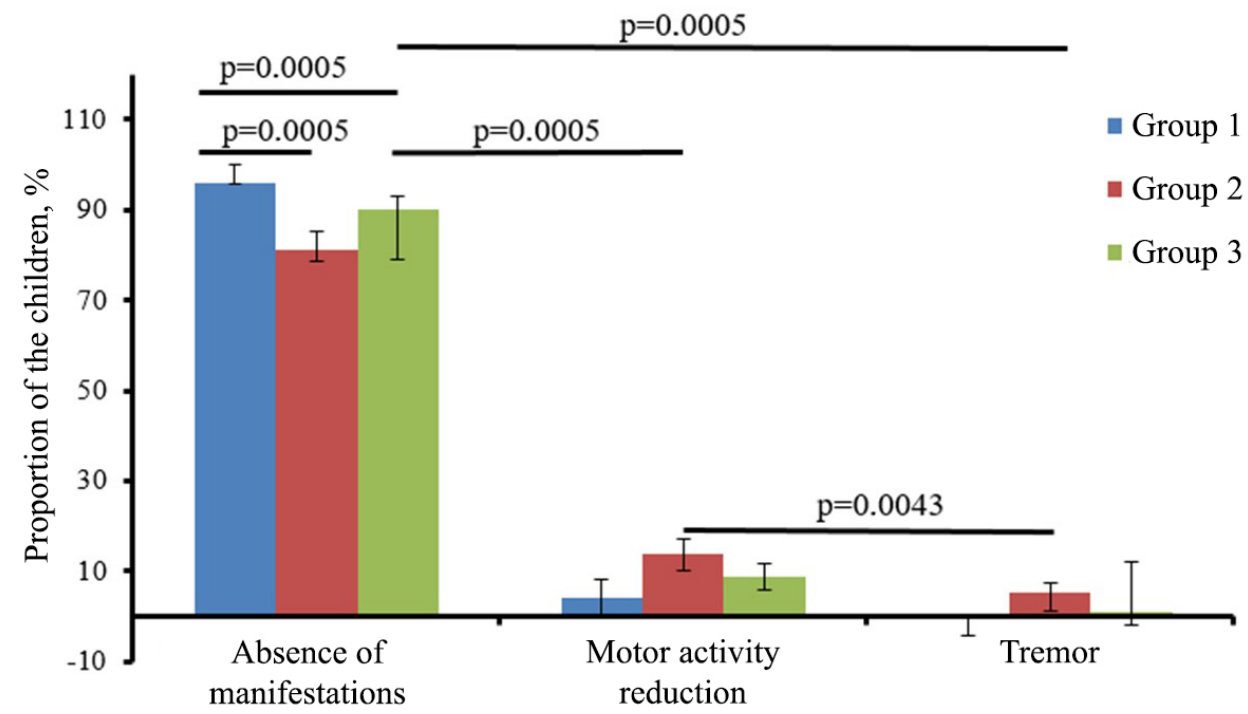

Figure 2. Distribution of newborns by clinical manifestations in identifying the minimum values of glycaemia in the studied groups. Note:* the assessment of statistical significance is presented according to the $\chi^{2}$ calculation.

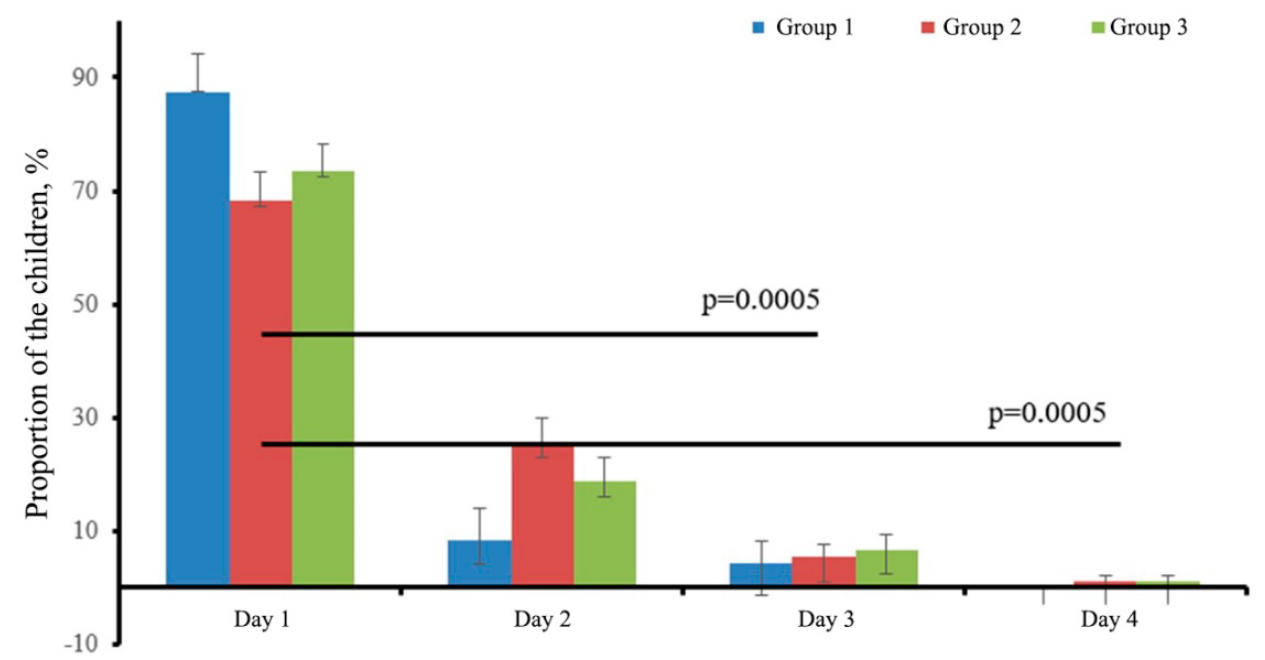

Figure 3. Distribution of newborns by age, taking into account the period of diagnosed hypoglycemia in the groups under consideration. Note:* the assessment of statistical significance is presented according to the $\chi 2$ calculation. 
and was accompanied by drastically reduced motor activity and reflexes. Clinical symptoms of hypoglycaemia were absent in $81.1 \%$ of the cases, while $13.7 \%$ of the children had such clinical manifestation as muscular hypotonia and tremor in extremities was registered in $5.3 \%$ of the cases (Figure 2). Distribution of the neonates according to their newly registered hypoglycaemia showed that low blood glucose levels were revealed during the 1 st day of life in $68.4 \%$ of the cases in this group, during the 2 nd day of life in $25.3 \%$ of the cases, during the 3 rd day of life in $5.3 \%$ of the cases and during the 4 th day of life in $1.1 \%$ of the cases (Figure 3). Analysis of minimum glycaemia levels revealed that the lowest blood glucose levels in $60 \%$ of the newborns were registered during the first day of life, in $31.6 \%$ during the 2 nd day of life, in $6.3 \%$ during the $3 \mathrm{rd}$ day of life and in $2.1 \%$ during the 4 th day of life. The treatment strategy involved parenteral glucose infusion (for $55.8 \%$ of the newborns in the studied group), peroral glucose administration (41.1\%) supplementary feeding with breast milk or infant formula (3.2\%). Glucose level normalisation (without consequent reduction) was achieved on the 1st day of life in $47.8 \%$, on the 2 nd day of life in $30 \%$, on the 3 rd day of life in $16.7 \%$, on the 4 th day of life in $4.4 \%$ and on the 5 th day of life in $1.1 \%$ of the children.

Children with IUGR amounted to $42.3 \%$ of the subjects, hypoglycaemia was revealed in 91 neonates (41.2\%, Figure 1). Among them $64.8 \%$ were male and $35.2 \%$ were female $(\mathrm{p}=0.0005)$. The median gestational age in the group was 38 weeks $[36 ; 69]$, the median body mass was 2270 [2015;2525]. The proportion of children with a body mass within the $3-10$ centile corridor was $24.2 \%$ and it was $75.8 \%$ for those below the $3 \mathrm{rd}$ centile. The minimum glucose level registered in the group was $0.9 \mathrm{mmol} / 1$ [2.0;2.4]. It is important that clinical symptoms of hypoglycaemia were absent in $90.1 \%$ of the cases (including the child with the lowest level of blood glucose). In the rest $0.9 \%$ of the cases, clinical manifestations of hypoglycaemia were tremor $(1.1 \%)$ and reduction of motor activity (8.8\%) (Figure 2). Age distribution among children with newly diagnosed hypoglycaemia was as follows: 1st day of life $-73.6 \%$, 2nd day of life $-18.7 \%$, 3rd day of life $-6.6 \%$, 4 th day of life $-1.1 \%$ (Figure 3). Dynamic evaluation of glycaemia within a 5-days period showed that the lowest indices of blood glucose were diagnosed in $63.7 \%$ of the cases during the 1 st day of life, in $20.9 \%$ during the 2 nd day of life, in $11 \%$ during the 3 rd day of life and in $4.4 \%$ during days $4-5$. Therapy against hypoglycaemia was performed via intravenous glucose infusion (61.7\%), administration of peroral glucose solution (33.0\%), supplementary feeding with breast milk or infant formula $(5.3 \%)$. Hypoglycaemia was reversed in $42.9 \%$ of the newborns during the 1 st day of life, in $20.9 \%$ during the 2 nd day of life, in $22 \%$ during the $3 \mathrm{rd}$ day of life, in $4.4 \%$ during the 4 th day of life, in $6.6 \%$ during the 5 th day of life and during days $5,8,18$ in $1.1 \%$ of the children.

\section{Discussion}

Hypoglycaemia detection rate in newborns with large body mass at birth was the lowest (in comparison to groups 2 and 3) amounting to $27 \%$ and being prevalent in male children. Maximum frequency of newly diagnosed hypoglycaemia in this risk group is registered during the 1st day of life (88\%). Thereat, minimum glycaemia values during 2 nd and 3rd days of life were registered to be $25 \%$ and $4 \%$, respectively. Glucose level stabilisation is achieved during the first day of life by the majority of newborns (67\% of the cases), however $33 \%$ achieve normoglycaemia during the 2 nd or 3 rd day of life.

Detection rate of hypoglycaemia in neonates born to mothers with diabetes mellitus was the highest and amounted to $44.6 \%$. The highest proportion of hypoglycaemia cases in this group of children was registered during the 1st day of life (68.4\%), minimum glycaemia values were most frequently observed during the 2 nd day of life $(32 \%$ of the newborns) and much less frequently during the 3rd or the 4th day of life ( $6.3 \%$ and $2.1 \%$ of the cases, respectively). Normalisation of glycaemia is achieved during the 1st day of life in one half of the cases (47.8\%), during the 2 nd day of life in $1 / 3$ of the children and within the period of 3 rd to 5 th days of life in $1 / 5$ of the children.

According to literature data, carbohydrate metabolism disorder in neonates born to mothers with diabetes mellitus is conditioned by transient compensatory hyperinsulinism, increased glucose tolerance and decreased glucagon secretion [11]. Therewith, hypoglycaemia in newborns in this group develops within the initial 6-12 hours after birth and is defined by the variant of the mother's diabetes mellitus. Thus, significant decrease in the child's glycaemia level against the background of the mother's diabetes mellitus type 1 is observed as early as within an hour after birth, while diabetes mellitus type 2 defines slower decrease in the child's glucose concentration with development of hypoglycaemia in 2 hours after birth or 2-3 hours after birth in cases of the mother's gestational diabetes mellitus. The term of the child's blood glucose value normalisation depends on a variety of factors and amounts to 48-72 hours after birth [12].

In neonates with IUGR, hypoglycaemia was registered in $41 \%$ of the cases with prevalence in male children. The lowest glycaemia indices, analogically to the previous groups, were registered during the first day of life. However, over $20 \%$ of the children showed decline in laboratory values for 4 days of the following observation. These changes in carbohydrate metabolism are determined by the absence of carbohydrate depot, inadequate glucogenesis, impaired peripheral insulin sensitivity and high energy expenditure in children with IUGR. Additional factors for increased hypoglycaemia duration are hypothermia and impaired thermoregulation due to underdevelopment of adipose tissue as well as hypoxia frequently observed in children of this group [13]. In $90.1 \%$ of the cases, glucose decrease was not accompanied by significant clinical manifestations including blood glucose values below $1 \mathrm{mmol} / \mathrm{l}$.

Hypoglycaemia in the studied newborns was not accompanied by clinical manifestations in $86.7 \%$ of the cases, significant symptoms in the studied cohort were not reported. In $89.2 \%$ of the children, progression of hypoglycaemia is asymptomatic, which confirms the importance and the necessity of glycaemia monitoring in the early neonatal period for timely and adequate therapy.

\section{Conclusion}

The conducted research has made it possible to present results of observation of 522 newborns with risk factors for hypoglycaemia (children with large birth weight, the mother's diabetes mellitus, IUGR) including analysis of hypoglycaemia detection rate within the first day of life, clinical and laboratory peculiarities of this condition as well as of hypoglycaemia dynamics in the early neonatal period and blood glucose stabilisation terms at different observation stages (the normoglycaemia achievement age). Detection rate of hypoglycaemia in the observed newborns was the highest in those who were born to mothers with diabetes mellitus (44.6\%) and with IUGR (41\%). Maximum frequency of newly-diagnosed hypoglycaemia is registered during the 1st day of life and is characterized by the absence of clinical symptoms.

\section{References}

1. Chandran S, Rajadurai VS, Alim A, Haium A, Hussain K (2015) Current perspectives on neonatal hypoglycemia, its management, and cerebral injury risk. J Res Reports Neonatol. 5: 17-30. 
2. Taranushenko TE, Kiseleva NG, Lazareva OV, Kalyuzhnaya II (2019) Hypoglycemia in newborns: literature review and case report. Endocrinology problems. Probl Endokrinol (Mosk) 65: 251-262 (in Russ). [Crossref]

3. Safina AI, Rybkina NL (2011) Transient hypoglycemia of newborns: etiology, diagnostic criteria, prevention and correction tactics in the early neonatal period. Practical Medic 53: 51-54 (in Russ).

4. Volodin NN (2019) Neonatology: a national guide. GEOTAR-Media. pp: 896 (in Russ).

5. Ayoub NI, Hanoudi BM, Naif M (2013) Evaluation of Maternal and Neonatal Risk factors for Neonatal Hypoglycemia. Iraqi J Comm Med 1: 13-18.

6. Ivanov DO, Shabalov NP, Petrenko Yu V (2015) Diagnostics and treatment of hypoglycemia in newborns: guidelines. w/e. pp: 58 (in Russ).

7. Kellams A, Harrel C, Omage S, Gregory C, Rosen-Carole C (2018) ABM Clinica Protocol \#3: Supplementary Feedings in the Healthy Term Breastfed Neonate, Revised 2017. Breastfeed Med 12: 188-198. [Crossref]
8. Queensland Clinical Guideline Supplement: Hypoglycaemia-newborn. 2019: 18. Available from: https://www.health.qld.gov.au/_data/assets/pdf_file/0043/881899/ghypogly.pdf

9. Committee on Fetus and Newborn, Adamkin DH (2011) Postnatal Glucose Homeostasis in Late-Preterm and Term Infants. Pediatrics. Pediatrics 127: 575-579. [Crossref]

10. Federal clinical guidelines (protocols) for the management of children with endocrine diseases. 2014: 442 (in Russ).

11. Ivanov DO (2011) Glucose metabolism disorders in newborns. SPb Publishing House NL. pp: 64 (in Russ).

12. Solodkova IV, Melnikova LN, Parshina NV, Petrenko Yu V, Fedoseeva TA, et al (2016) Children from mothers with diabetes mellitus. Diabetes mellitus in newborns: clinical guidelines (draft). w/e, pp: 22 (in Russ).

13. Nagaeva EV, Shiriaeva TL (2010) Intrauterine programming of hormonal and metabolic processes and intrauterine growth retardation syndrome. Problems Endocrinol 56: 32 40 (in Russ).

Copyright: $(2021$ Taranushenko TE. This is an open-access article distributed under the terms of the Creative Commons Attribution License, which permits unrestricted use, distribution, and reproduction in any medium, provided the original author and source are credited. 